\title{
System der Blütenpflanzen - Neue Zugehörigkeiten, neue Namen
}

\author{
Clemens Bayer, Theodor C.H. Cole, Hartmut H. Hilger
}

\begin{abstract}
Since the introduction of molecular analyses in the field of phylogenetic reconstructions, great progress has been achieved in botanical systematics. As a consequence of the recent findings, a number of changes in the circumscription and naming of several plant families were found to be necessary. This paper provides a brief introduction into the APG system, which became a standard in botany.
\end{abstract}

\section{Zusammenfassung}

Seit versucht wird, die Stammesgeschichte der Blütenpflanzen anhand molekularer Analysen zu rekonstruieren, hat die botanische Systematik erhebliche Fortschritte zu verzeichnen. Als Konsequenz aus den neuen Erkenntnissen ergaben sich Änderungen in der Umgrenzung und Benennung von einigen Pflanzenfamilien. Als Standard etabliert sich zunehmend das so genannte APG-System, das hier kurz vorgestellt wird.

\section{Phylogenie und Benennung}

Die wissenschaftliche Botanik bemüht sich um ein System, das die Stammesgeschichte (Phylogenie) und damit die natürlichen Verwandtschaftsbeziehungen zwischen den Pflanzengruppen möglichst genau widerspiegelt. Seit der Einführung molekularer Techniken und der Anwendung computergestützter Auswertungsverfahren zur Rekonstruktion der Phylogenie sind wir diesem Ziel in den letzten drei Jahrzehnten wesentlich näher gekommen. Insbesondere gelang es, verschiedene „künstliche“ Pflanzengruppen zu identifizieren, die aufgrund oberflächlicher Ähnlichkeiten oder mangels überzeugenderer Alternativen zusammengestellt worden waren. Mit Hilfe der neuen Methoden konnten die Verwandtschaftsverhältnisse für die meisten solcher Gruppen erstmals aufgedeckt werden. In der Folge war und ist es unvermeidlich, dass manche Pflanzenfamilien, Ordnungen, Gattungen etc. neu umschrieben, teils auch neu benannt werden mussten, um der verbesserten Kenntnis der Verwandtschaftsverhältnisse Rechnung zu tragen: Schließlich will man nicht mit veralteten Namen arbeiten, von denen man genau weiß, dass sie für künstliche Gruppen stehen, obwohl längst eine nachvollziehbare Zuordnung zu einer stammesgeschichtlich begründeten, natürlichen Gruppe vorliegt.

Nun stößt es bei Praktikern wie Gärtnern und anderen Pflanzenfreunden oft auf Unverständnis oder gar Ablehnung, wenn Pflanzennamen ge- ändert werden. Viele sind skeptisch, ob das nötig sei und ob die neuen Namen dann länger gültig bleiben als die alten. Selbstverständlich ist Stabilität auch für moderne Systematiker ein äußerst wichtiges Ziel. Seit Menschen Pflanzen benennen, hat es aber immer Namens-Änderungen gegeben und wird es auch in Zukunft geben, weil es immer neue Erkenntnisse gibt. Andererseits wäre es falsch (und wie in anderen Zweigen der Wissenschaften undenkbar), den neuen Erkenntnissen nicht Rechnung zu tragen.

\section{Beispiel Malvaceae}

Um zu illustrieren, wie es zu solchen Umbenennungen/Zuordnungen kommen kann, soll im Folgenden das Beispiel der Malvengewächse kurz erläutert werden. Bis vor wenigen Jahren waren von den meisten Botanikern außer den Malvaceae (den eigentlichen Malven mit Hibiscus, Althaea, Abutilon etc.) noch die Linden-, Wollbaum- und Kakaogewächse (Tiliaceae, Bombacaceae und Sterculiaceae) als eigenständige Familien akzeptiert; manche Autoren unterschieden sogar noch mehr Familien. Heute wird nicht nur in der Fachliteratur, sondern auch in Lehrbüchern und auf Pflanzenetiketten in Bota-

Abb. 1 (S. 111): Verteilung von Vertretern der ehemaligen Familien Tiliaceae (rot), Sterculiaceae (blau), Bombacaceae (grün) und Malvaceae (gelb) in einer Analyse von DNASequenzdaten (BAYER et al. 1999) zur Veranschaulichung, dass diese keine natürlichen Gruppen darstellten. 


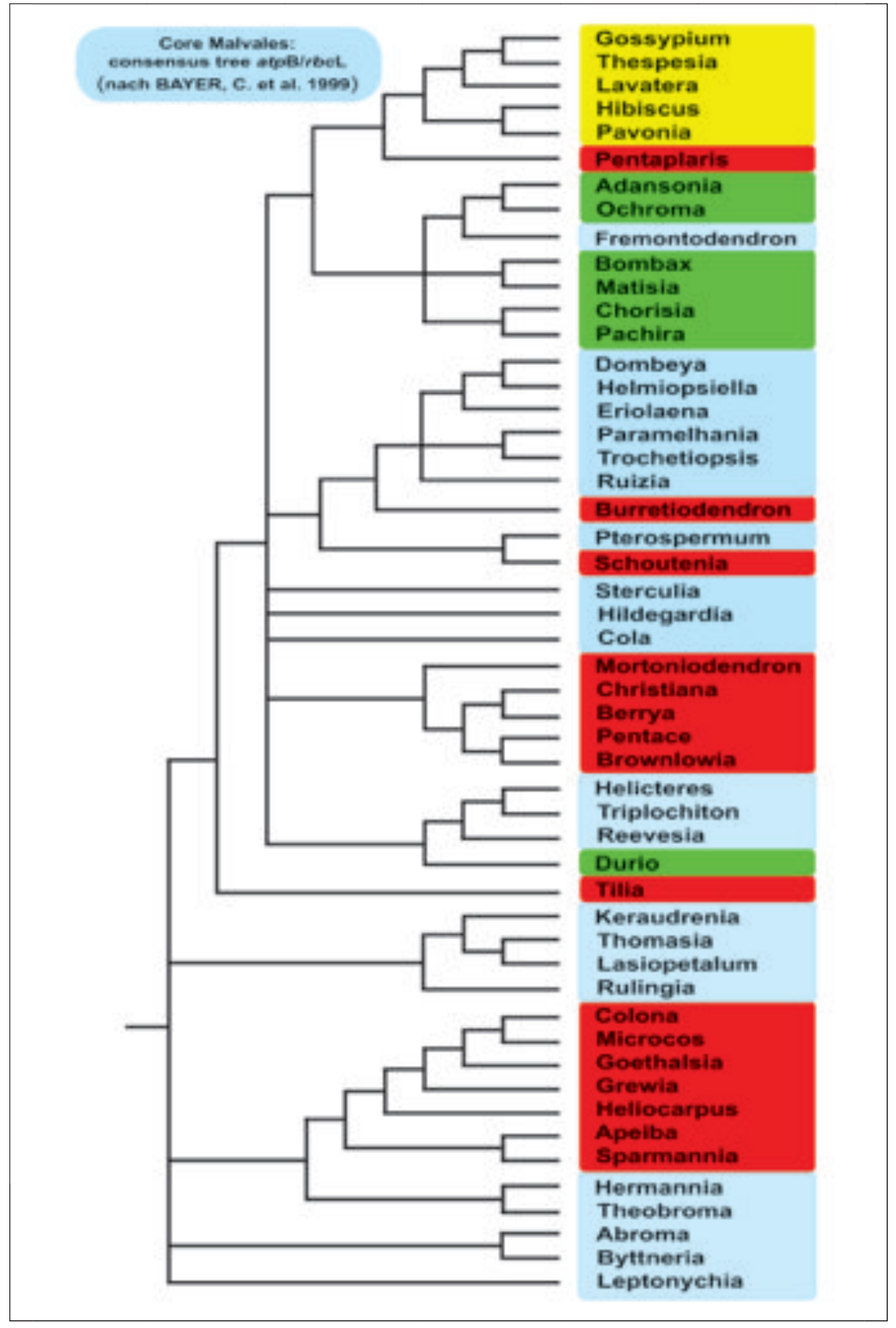


nischen Gärten, nur noch von einer einzigen, um die übrigen drei erweiterten Familie Malvaceae gesprochen, so dass beispielsweise Linde, Kapok-, Kakao- und Cola-Baum jetzt zu den Malvengewächsen gerechnet werden.

Schon seit langer Zeit war unklar und uneindeutig, wie man denn die früheren Familien Tiliaceae, Sterculiaceae, Bombacaceae und Malvaceae unterscheiden und gegeneinander abgrenzen kann, und manche Autoren hatten darauf hingewiesen, dass es sich nicht um natürliche Gruppen und eine sinnvolle Gliederung handeln könnte. Bestätigt wurde dies durch eine Analyse struktureller Merkmale, die allerdings keine Alternative zur traditionellen Unterteilung aufzeigte (JUDD \& MANCHESTER 1997). Erst mit dem Einsatz molekularer Methoden wurde das Bild so klar, dass eine Neugliederung möglich wurde: Auswertungen von Sequenzanalysen zweier Chloroplasten-Gene ergaben Gruppen, die zwar nicht mit der früheren Gliederung in vier Familien, dafür aber mit der Verteilung morphologischer Merkmale zusammenpassten (BAYER et al. 1999).

Nachdem damit klar war, dass die vier Familien in ihrer traditionellen Umschreibung künstlich und nicht haltbar waren, wurde als Alternative vorgeschlagen, sie in einer einzigen Familie Malvaceae unterzubringen und diese in neun Unterfamilien zu gliedern (BAYER et al. 1999). Andere mögliche Konsequenzen wären gewesen, diese Gruppen als Triben oder aber als Familien zu behandeln, wie jüngst vorgeschlagen wurde (HEYwOOD et al. 2007). Dies hätte aber u. a. den Nachteil, dass man teils neue, teils missverständliche Familiennamen verwenden müsste (so würden z. B. die Tiliaceae nicht den gewohnten Umfang behalten, sondern auf drei Gattungen reduziert). Inzwischen hat sich der Ansatz einer erweiterten Familie Malvaceae mit neun Unterfamilien weitgehend durchgesetzt.

\section{Das APG-System}

Im Folgenden soll das aktuelle System der Blütenpflanzen bis zum Niveau der Familien kurz erläutert werden, wobei besonders die wichtigsten Änderungen gegenüber älteren Systemen dargestellt werden. Die gute Nachricht vorab ist, dass sich der größte Teil der bekannten Familiennamen nicht geändert hat. Die Änderungen konzentrieren sich vor allem auf Familien, deren Umgrenzung schon früher umstritten war oder für die bezweifelt wurde, dass sie natürliche Verwandtschafts-Gruppen darstellen.

Für diejenigen Familien, deren Umfang und/oder Stellung sich aufgrund molekularer Befunde geändert haben, kann man sagen, dass sich ein hohes Maß an Stabilität abzeichnet. Die erste umfangreiche Analyse zur molekularen Phylogenie der Pflanzen, die von 42 (!) Botanikern gemeinsam veröffentlicht wurde (CHASE et al. 1993), hat neben breiter Zustimmung auch Unverständnis und Ablehnung bis hin zu Polemik ausgelöst, was sich aber auf wenige überraschende Befunde bezog. In der Folge und bis heute wurden aber unzählige weitere und genauere Untersuchungen durchgeführt, welche die meisten auch der gewöhnungsbedürftigen Ergebnisse von CHASE et al. (1993) bestätigten. Deshalb kann man davon ausgehen, dass die Anzahl der nötigen Namensänderungen zumindest auf Familienniveau geringer bleibt als in der Vergangenheit. Insofern ist die Umsetzung der Korrekturen auch als Beitrag zur Stabilität der Pflanzennamen zu sehen.

Die zahlreichen Ergebnisse der Versuche, die Stammesgeschichte der Pflanzen mit Hilfe molekularer Methoden zu rekonstruieren, wurden von einer Arbeitsgruppe namens "Angiosperm Phylogeny Group" (APG) zu einem phylogenetischen System der Pflanzenfamilien zusammengeführt (APG 1998, aktualisiert als APG II, 2003, und APG III, 2009), das heute als wissenschaftlicher Standard weitgehend akzeptiert wird.

Ganz summarisch kann man sagen, dass die untersten Äste des phylogenetischen Baumes die Amborellaceae, Nymphaeales, Austrobaileyales und Chloranthales umfassen, alles Gruppen, die auch schon früher immer wieder als „ursprünglich" angesehen wurde. Dies gilt auch für die folgende Gruppe der Magnoliiden (mit Canellales, Piperales, Laurales und Magnoliales). Dann erst, also als Seitenast innerhalb der Zweikeimblättrigen und nicht etwa als deren Schwestergruppe,

Abb. 2: Phylogenetisches System nach APG 


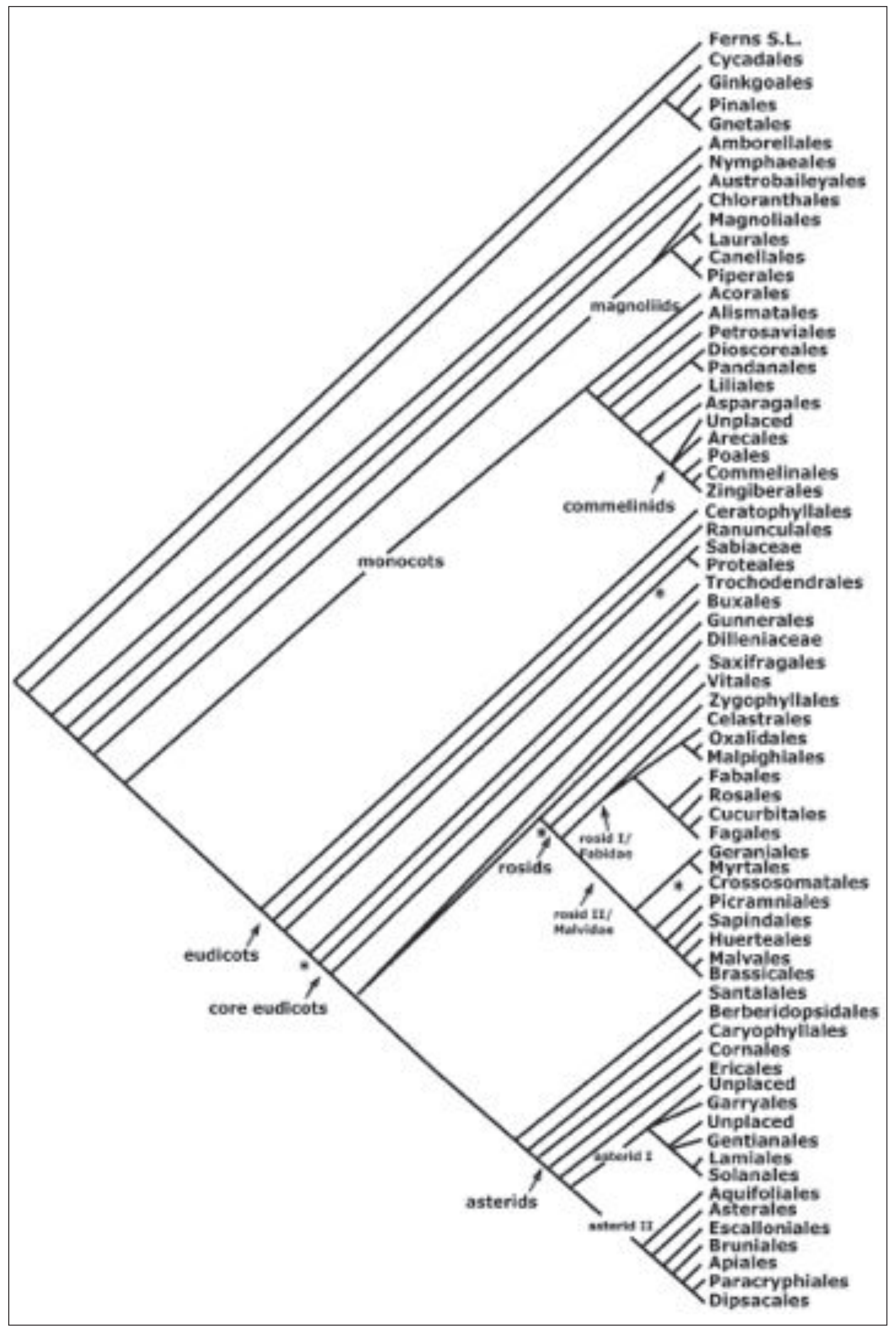



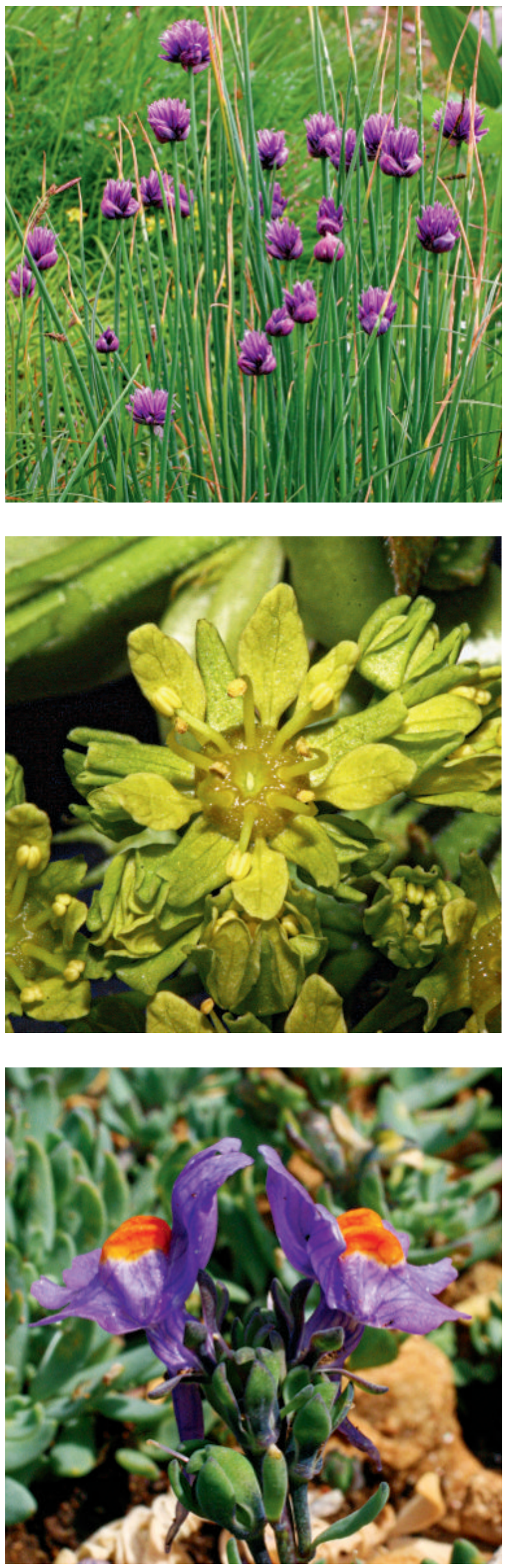

wie es frühere Systeme nahegelegt hatten, zweigen die Monocotylen (Einkeimblättrigen) ab. Sie sind klar abgegrenzt und bilden eine natürliche Einheit; an ihrer Basis steht der Kalmus (Acorus). Die folgenden Gruppen werden (teils unter Ausschluss der Hornkräuter, Ceratophyllaceae) als „die eigentlichen Zweikeimblättrigen“" (Eudicots) bezeichnet. An ihrer Basis stehen die Hahnenfußartigen (Ranunculales), die Proteales (wo Lotosblumen und Platanen direkt nebeneinander stehen, was anfangs für Irritationen sorgte), Trochodendrales und Buxales. In der verbleibenden, größten Gruppe der Zweikeimblättrigen stehen die Gunnerales und Saxifragales an der Basis, die übrigen werden in Rosiden (aus Fabidae und Malvidae) und Asteriden (Lamiidae und Campanulidae) gegliedert (APG III, siehe Abb. 2).

\section{Wichtige Änderungen}

In Umfang und Benennung folgender bekannter Familien ergaben sich gegenüber früherer Systeme größere Änderungen (siehe auch Schroeder 2007, Callauch 2008, Hilger et al. 2010):

Die Liliaceae umfassten früher einen großen Teil der einkeimblättrigen Pflanzen. Die meisten davon (sowie die Orchideen) stehen heute in der Ordnung Asparagales. Dort werden u. a. die früheren Agavaceae und die Hyacinthaceae heute zu den Asparagaceae gestellt; die Alliaceae und Agapanthaceae wurden in die Amaryllidaceae überführt.

Für die Euphorbiaceae zeichnet sich ab, dass die einzelnen Linien wohl nicht in einer einzigen Familie untergebracht werden können, so hat man u. a. die Phyllanthaceae als eigene Familie abgetrennt, doch sind die Arbeiten noch nicht ganz abgeschlossen.

Ganz aufgelöst wurden die Flacourtiaceae, ein großer Teil der Gattungen (darunter die bekannteren wie Azara und Idesia) wurde zu den Salicaceae gestellt, die übrigen zu den Achariaceae.

Abb. 3 (oben): Allium schoenoprasum, früher Alliaceae, heute Amaryllidaceae.

Abb. 4 (Mitte): Acer platanoides, früher Aceraceae, heute Sapindaceae.

Abb. 5 (unten): Linaria alpina, früher Scrophulariaceae, heute Plantaginaceae. 
Die Fabaceae (Leguminosen, Schmetterlingsblütler) umfassen heute, wie auch schon bei vielen älteren Autoren und im angelsächsischen Sprachgebrauch üblich, auch die früheren Caesalpiniaceae und Mimosaceae.

Dass die Malvaceae jetzt auch die ehemaligen Bombacaceae, Sterculiaceae und Tiliaceae einschließen, wurde eingangs erwähnt.

Wie schon länger bekannt war, sind die Rosskastanien und Ahorn-Arten eng mit den Sapindaceae verwandt. Heute werden die Aceraceae und Hippocastanaceae konsequenter Weise zu den Sapindaceae gestellt.

Nachdem die Primulaceae zunächst auf wenige Gattungen reduziert wurden, umfasst die Familie nach APG III (2009) jetzt wieder die ausgegliederten Gruppen, indem sie um die früheren Myrsinaceae, Theophrastaceae und Maesaceae erweitert wurde.

Innerhalb der Caryophyllales wurden die Chenopodiaceae zu den Amaranthaceae gestellt.

Die Scrophulariaceae umfassen jetzt nur noch wenige Gattungen, darunter auch Buddleja. Die meisten Gattungen der ehemaligen Rachenblütler (z. B. Veronica, Digitalis, Mimulus, Antirrhinum) stehen heute bei den Plantaginaceae, andere in den Calceolariaceae. Halb oder voll parasitische Gattungen wie Klappertopf, Läusekraut, Augentrost und Verwandte werden dagegen zu den Orobanchaceae gestellt. Paulownia steht jetzt in einer eigenen Familie.

Viele Gattungen wie Clerodendron, Vitex oder Callicarpa, die früher zu den Verbenaceae gerechnet wurden, stehen jetzt bei den Lamiaceae.

Die ehemaligen Asclepiadaceae sind heute nur noch eine Unterfamilie der Apocynaceae.

Auch im Bereich der Caprifoliaceae gab es umfangreiche Änderungen, beispielsweise werden Sambucus und Viburnum heute den Adoxaceae zugeordnet.

Abb. 6 (oben): Paederota bonarota, früher Scrophulariaceae, heute Plantaginaceae.

Abb. 7 (Mitte): Melampyrum cristatum, früher Scrophulariaceae, heute Orobanchaceae.

Abb. 8 (unten): Vitex agnus-castus, früher Verbenaceae, heute Lamiaceae.
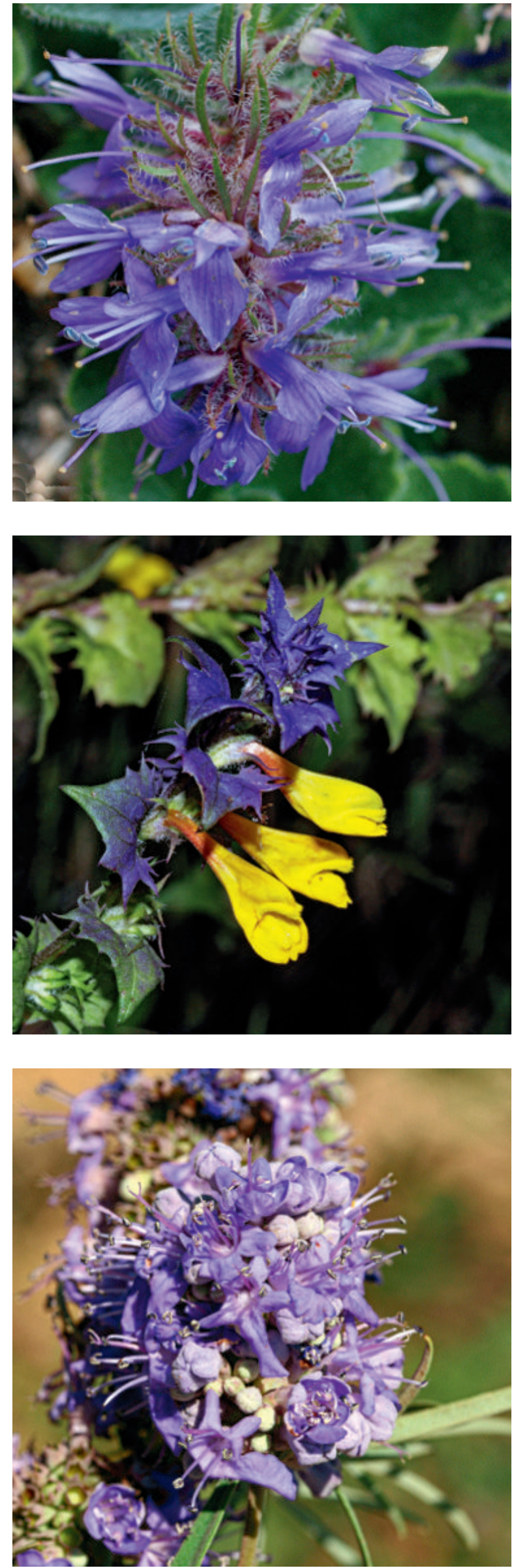

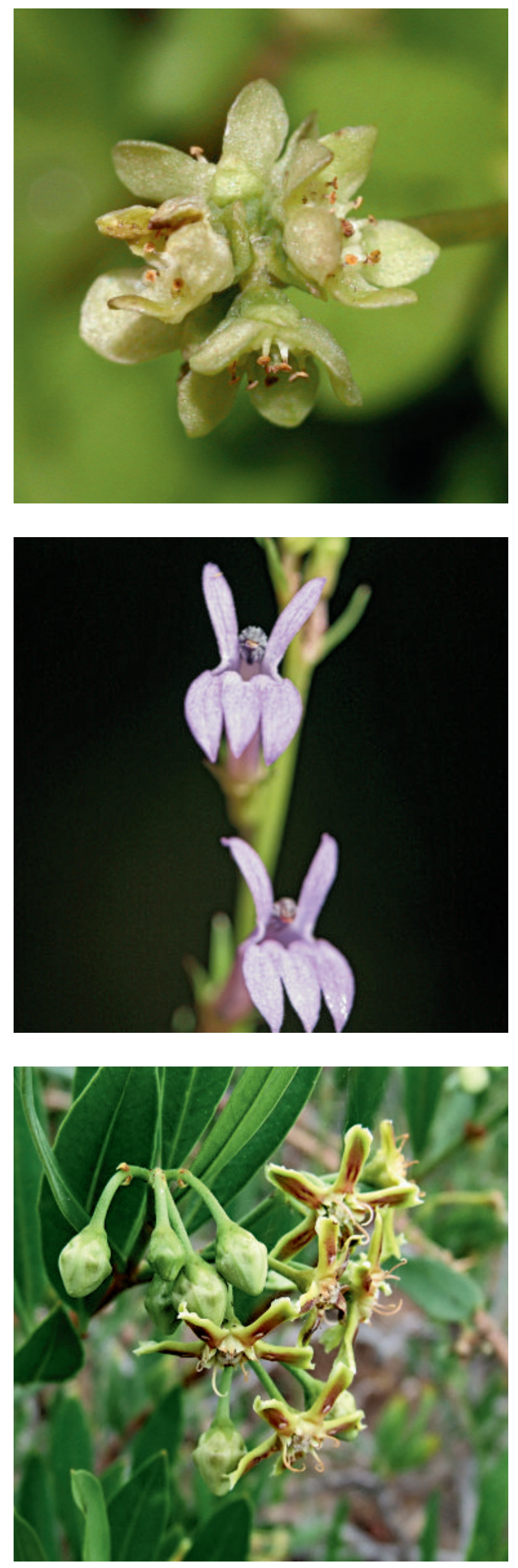

Auch die Lobeliaceae sind keine eigene Familie mehr, sondern gehören jetzt zu den Campanulaceae.

\section{Empfehlungen}

Neu gewonnene Erkenntnisse zu den Verwandtschaftsbeziehungen der Blütenpflanzen führen zwangsläufig zu Veränderungen der wissenschaftlichen Pflanzennamen. Diese finden mit einer gewissen zeitlichen Verzögerung Eingang in unterschiedlichste Bereiche von Forschung und Praxis. Im gärtnerischen Bereich wird man teils mit aktuellen, teils mit überholten Namen und Zugehörigkeiten konfrontiert. Vielfach ist es einfach zu aufwendig, beispielsweise alle Pflanzenschilder zu erneuern, so dass nur die neuen Schilder die aktualisierte Benennung aufweisen. Im Zweifelsfalle muss man entsprechend moderne Nachschlagewerke konsultieren. Hierfür gibt es außer gedruckten Werken wie KUBITZKIs "Families and genera of vascular plants" (seit 1990) oder das kompakte Plant-Book (MABBERLEY 2008) vor allem die hilfreichen und frei zugänglichen Seiten im Internet, die laufend aktualisiert werden. Empfohlen sei neben der "Angiosperm phylogeny website" von PETER STEVENS (seit 2001) für Familien und höhere Kategorien die GRIN-Seiten (Germplasm resources information network) des US-amerikanischen Landwirtschaftsministeriums USDA. Als Überblick und Lernhilfe steht im Internet auch ein kostenloses „Poster - Stammbaum der Angiospermen“ bzw. "Angiosperm Phylogeny Poster" auf Deutsch und Englisch zur Verfügung (COle \& Hilger 2010ff).

\section{Dank}

Die Autoren danken Peter F. Stevens, der freundlicherweise die Vorlage für Abb. 2 zur Verfügung gestellt hat, REINHARD JAHN und MAXIMilian Weigend, die uns Pflanzenbilder zum

Abb. 9 (oben): Adoxa moschatellina, heute zusammen mit Sambucus und Viburnum in den Adoxaceae.

Abb. 10 (Mitte): Lobelia urens, früher Lobeliaceae, heute Campanulaceae.

Abb. 11 (unten): Periploca laevigata, früher Asclepiadaceae, heute Apocynaceae. 
Abdruck überlassen haben, und ULRIKE BRUNKEN, die hilfreiche Verbesserungsvorschläge zum Manuskript beigetragen hat.

\section{Literatur}

APG 1998: An ordinal classification for the families of flowering plants. - Ann. Missouri Bot. Gard. 85: 531-553. APG II. 2003. An update of the Angiosperm Phylogeny Group classification for the orders and families of flowering plants: APG II. - Bot. J. Linn. Soc. 141: 399-436. APG III. 2009. An update of the Angiosperm Phylogeny Group classification for the orders and families of flowering plants: APG III. - Bot. J. Linn. Soc. 161: 105-121. Bayer, C., Fay, M. F., De Bruijn, A. Y., Savolainen, V., Morton, C. M., Kubitzki, K., Alverson, W. S. \& CHASE, M.W. 1999: Support for an expanded family concept of Malvaceae within a recircumscribed order Malvales: a combined analysis of plastid $a t p B$ and $r b c L$ DNA sequences. - Bot. J. Linn. Soc. 129: 267-303.

Callauch, R. 2008: Aktuelle Veränderungen bei den Pflanzennamen. - Gartenpraxis 12: 50-54.

Chase, M. W., Soltis, D. E., Olmstead, R. G., Morgan, D., Les, D. H., Mishler, B. D., Duvall, M. R., Price, R. A., Hills, H. G., QiU, Y.-L., Kron, K. A., Rettig, J. H., Conti, E., Palmer, J. D., Manhart, J. R., Sytsma, K. J., Michaels, H. J., Kress, W. J., Karol, K.G., Clark, W. D., Hedren, M., Gaut, B. S., Jansen, R. K., Kim, K.-J., Wimpee, C.F., Smith, J. F., Furnier, G.R., Strauss, S. H., Xiang, Q.-Y., Plunkett, G. M., Soltis, P.S., Swensen, S.M., Williams, S. E., GadeK, P. A., Quinn, C. J., Eguiarte, L. E., GolenberG, E., Learn, G. H., Graham, S. W., Barrett, S. C. H., Dayanandan, S. \& AlberT, V. A. 1993. Phylogenetics of seed plants an analysis of nucleotide sequences from the plastid gene rbcL. - Ann. Missouri Bot. Gard. 80: 528-580.

Heywood, V. H., Brummitt, R. K., Culham, A. \& SEBERG, O. 2007: Flowering plant families of the world. - Kew.

Hilger, H.H., Cole, T.C.H. \& WinK, M. 2010: Neue Namen für altbekannte Arznei- und Giftpflanzen. Deutsche Apotheker-Zeitung 150: 3114-3123.

JUdD, W. S. \& MANCHESTER, S. R. 1997. Circumscription of Malvaceae (Malvales) as determined by a preliminary cladistic analysis of morphological, anatomical, palynological, and chemical characters. - Brittonia 49: 384-405. KuBITZKI, K. (Hrsg.) 1990 ff.: The families and genera of vascular plants. - Heidelberg etc.

Mabberley, D. J. 2008: MabBerley's plant book. A portable dictionary of plants, their classification and uses, 3. Aufl. - Cambridge.

Abb. 12 (oben): Agave attenuata, früher Agavaceae, heute Asparagaceae.

Abb. 13 (unten): Agapanthus africanus, früher Agapanthaceae, heute Amaryllidaceae.
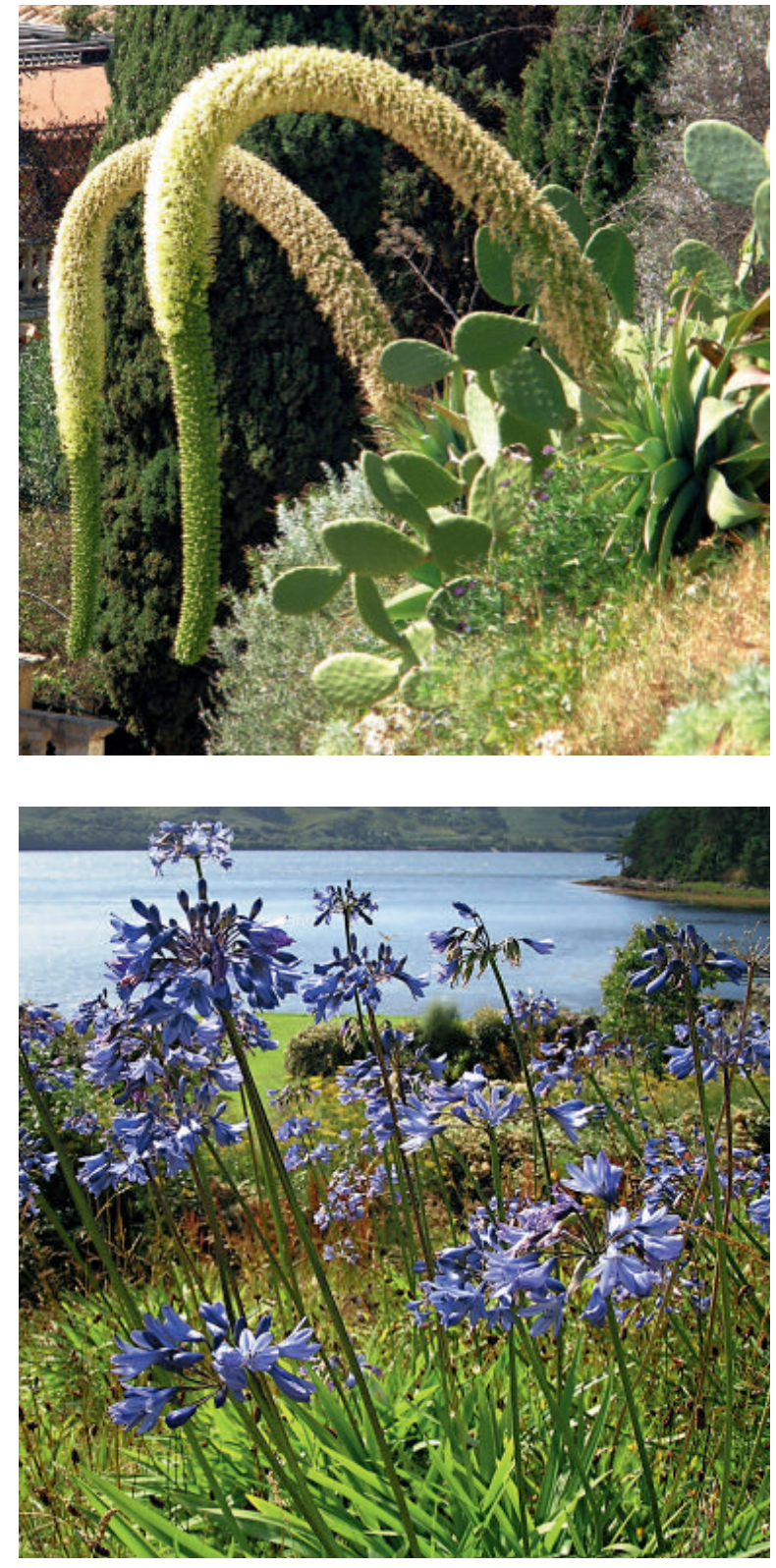

SCHRÖDER, F.-G. 2007. Neuerungen bei der Systematik der Gehölze. - Mitt. Dtsch. Dendrol. Ges. 92: 11-20.

\section{Internetseiten}

Cole, T. C. H. \& Hilger, H. H. 2010 ff.: Angiosperm phylogeny poster - Flowering plant systematics. http://www.biologie.fu-berlin.de/sysbot/poster/poster1.pdf

STEVENS, P. F. 2001 ff.: Angiosperm phylogeny website (APweb). - http://www.mobot.org/MOBOT/research/APweb/

USDA, ARS, National Genetic Resources Program. Germplasm Resources Information Network (GRIN). National Germplasm Resources Laboratory, Beltsville, Maryland. - http://www.ars-grin.gov/cgi-bin/npgs/html/ paper.pl?language $=$ de. 